\title{
Atomistic calculations of lattice constants of mullite with its compositions
}

\author{
Jen-Chang Chen ${ }^{\mathrm{a}}$, Chuin-Shan Chen ${ }^{\mathrm{a}, *}$, Hartmut Schneider ${ }^{\mathrm{b}}$, \\ Chia-Ching $\mathrm{Chou}^{\mathrm{a}}$, Wen-Cheng J. Wei ${ }^{\mathrm{c}}$ \\ ${ }^{a}$ Department of Civil Engineering, National Taiwan University, Taipei, Taiwan \\ ${ }^{\mathrm{b}}$ Institute of Crystallography, University of Cologne, Köln, Germany \\ ${ }^{\mathrm{c}}$ Department of Materials Science and Engineering, National Taiwan University, Taipei, Taiwan
}

Available online 16 April 2007

\begin{abstract}
We calculate lattice constants $a$ and $b$ of mullite with its compositions $\left(\mathrm{Al}_{2}\left[\mathrm{Al}_{2+2 x} \mathrm{Si}_{2-2 x}\right] \mathrm{O}_{10-x}\right)$ using the constant stress molecular dynamics (MD) method. The Matsui's and Winkler's interatomic models for aluminosilicate are adapted. Two sets of mullite models are considered, one with a random distribution of oxygen vacancies and Al/Si substitutions and the other with a partially ordered $\mathrm{Al}$ occupancy at the $\mathrm{T}^{*}$ sites. All the simulation results predict the trend observed in experiments in which the lattice constant $a$ increases while $b$ decreases when the $x$-value increases. The expansion mechanism induced by Al/Si substitutions is supported by bond length analysis. The reduction of lattice constant $b$ is supported by the locally anisotropic atom displacement in the $\mathbf{a}$ and $\mathbf{b}$ directions sensed by the oxygen vacancies. We conclude that the causes of lattice expansion and reduction are tightly interwoven with the coexistence of vacancies and $\mathrm{Al} / \mathrm{Si}$ substitutions and their inherent influence on atom displacements. (C) 2007 Elsevier Ltd. All rights reserved.
\end{abstract}

Keywords: Mullite; Defects; Molecular dynamics

\section{Introduction}

Mullite has outstanding creep resistance, low thermal expansion, low thermal conductivity, high-temperature strength and good chemical stability. These properties make it a strong candidate for structural and high-temperature applications. ${ }^{1}$ The crystalline structure of mullite consists of edge-sharing $\mathrm{AlO}_{6}$ octahedral chains interconnected by $(\mathrm{Si}$ and $\mathrm{Al}) \mathrm{O}_{4}$ tetrahedra. In general, mullite compositions can be described as $\mathrm{Al}_{2}\left[\mathrm{Al}_{2+2 x} \mathrm{Si}_{2-2 x}\right] \mathrm{O}_{10-x}$ in which $x$ is the number of oxygen vacancy per unit cell, the $\mathrm{Al}$ and $\mathrm{Si}$ atoms within the bracket are tetrahedral bound, and the other $\mathrm{Al}$ atoms are octahedral bound. $^{2}$ As the $x$-value increases, the oxygen vacancies and the aluminum contents increase. Although mullite normally forms a solid solution of aluminosilicate with the $x$-value ranging between 0.25 ( 72 wt. $\% \quad \mathrm{Al}_{2} \mathrm{O}_{3}$, i.e., $3 \mathrm{Al}_{2} \mathrm{O}_{3}-2 \mathrm{SiO}_{2}=3 / 2$ mullite) and 0.40 (78 wt. $\% \mathrm{Al}_{2} \mathrm{O}_{3}$, i.e., $2 \mathrm{Al}_{2} \mathrm{O}_{3}-\mathrm{SiO}_{2}=2 / 1$ mullite), the existence of $x$ in the range of $0.18-0.88$ has been reported..$^{3,4}$

\footnotetext{
* Corresponding author. Tel.: +886 23366 4275; fax: +886223672558 E-mail address: dchen@ntu.edu.tw (C.-S. Chen).
}

The crystal structure of mullite displays a near-tetragonal atom arrangement in the crystallographic $\left(\begin{array}{lll}0 & 0 & 1\end{array}\right)$ plane. Such arrangement would logically imply that its $a$ and $b$ lattice constants should both increase or decrease in a similar fashion with the $x$-value. However, experimental observations indicate an anomaly. ${ }^{\mathrm{a}, 2,5-8}$ Cameron $^{2,5}$ found that the cell dimension in the a direction expands linearly while that in the $\mathbf{b}$ direction slightly contracts with increasing $x$-value of mullite. Based on extrapolation, Cameron estimated that the crossing point of lattice constants $a$ and $b$ was about at $x=0.67 .^{2}$ To explain this anomalous behavior of lattice constants in the $\left(\begin{array}{ll}0 & 0\end{array}\right)$ plane, Fischer and Schneider ${ }^{8}$ proposed two possible mechanisms: one was a contraction mechanism due to oxygen vacancies and the other was an expansion mechanism due to the substitution of $\mathrm{Si}^{4+}$ with $\mathrm{Al}^{3+}$. They further hypothesized that the anomaly might be reasoned by the possibility of structurally anisotropic distribution of vacancies since randomly distributed vacancies and substitutions would likely have similar expansion-reducing effects in both the $\mathbf{a}$ and $\mathbf{b}$ directions.

\footnotetext{
a The terms "anomaly" and "anomalous" used herein are intended to highlight the unexpected lattice trend observed in experiments.
} 
Table 1

Potential parameters and atom charges for the Winkler's potential

\begin{tabular}{|c|c|c|c|c|c|}
\hline Atom pair & $A\left(\mathrm{~kJ} \mathrm{~mol}^{-1}\right)$ & $B\left(\AA^{-1}\right)$ & $\alpha(\AA)$ & $C\left(\mathrm{~kJ} \mathrm{~mol}^{-1} \AA^{6}\right)$ & Charge $(e)$ \\
\hline $\mathrm{Al}-\mathrm{O}$ & 826542.9399 & 4.6620 & 0.0000 & 7052.2141 & $\mathrm{Al}:+1.8000$ \\
\hline $\mathrm{Si}-\mathrm{O}$ & 1737092.5134 & 4.8733 & 0.0000 & 12884.4236 & $\mathrm{Si}:+2.4000$ \\
\hline $\mathrm{O}-\mathrm{O}$ & 133995.7629 & 2.7601 & 0.0000 & 16884.8750 & $\mathrm{O}:-1.2000$ \\
\hline
\end{tabular}

The anomaly of lattice constants of mullite and the aforementioned, intuitively sound mechanisms have motivated us to investigate the subject through the venue of molecular dynamics (MD) simulation. MD simulation provides a direct and powerful route to study the movement of atoms, and consequently the collective mechanisms induced by this movement. The movement of atoms is governed by classical mechanics. The interactions between atom species are described by empirical force fields. The initial atom positions are often obtained from crystallographic data. ${ }^{\mathrm{b}} \mathrm{MD}$ then calculates atom positions and velocities using the finite difference method with a small timestep, normally $0.5-1$ fs. Thermodynamics properties are related to atom positions and velocities through the machinery of statistical ensembles. MD simulation often provides a theoretical basis for understanding experimental results in materials science. For the subject related to mullite, Winkler et al. ${ }^{9}$ performed MD on amorphous aluminosilicate $\left(\mathrm{Al}_{2} \mathrm{O}_{3} \cdot 2 \mathrm{SiO}_{2}\right)$ at the temperature higher than the melting point to study the structure and selfdiffusivity at the melting state. Lacks et al. ${ }^{10}$ applied MD on the sillimanite and mullite $(x=0.26)$ to study the anomaly of heat capacity of mullite.

The objective of this study is to shed light on the causes of lattice anomaly of mullite using MD simulation. To this end, proper derivations of the mullite structure from sillimanite are discussed. Two well-known interatomic potential models for aluminosilicate are adapted and their influence on lattice constant prediction is studied. Extensive analysis of simulation results is conducted to probe the possible mechanisms responsible for lattice contraction and expansion of mullite.

\section{Simulation methods}

We applied the Nosé-Hoover constant stress ensemble $(N \sigma T)$ on a mullite $18 \times 18 \times 6$ supercell using the DL_POLY package (Version 2.15). ${ }^{11}$ The NoT ensemble modifies the equations of motion with constraints so that the system keeps constant number of atoms $(N)$, constant stress $(\sigma)$, and constant temperature $(T)$ during the simulation. This ensemble allows anisotropic changes of cell size and shape. ${ }^{12,13}$ We used Melchionna's version of Hoover dynamics. ${ }^{13}$ Both the thermostat and barostat were considered at the same equations of motion. The preset temperature affects the frictional coefficient $\zeta$ and the preset pressure controls the piston variable $\eta$. In this study, the simulation temperature was set at $25^{\circ} \mathrm{C}(298 \mathrm{~K})$ and the external pressure was set at $1 \mathrm{~atm}(101.325 \mathrm{kPa})$. The relaxation time for thermostat and barostat was 0.5 and $0.8 \mathrm{ps}$, respectively.

\footnotetext{
${ }^{\mathrm{b}}$ Nevertheless, the atom coordinates of mullite used in MD cannot be obtained directly from crystallographic data, as shall be explained in the next section.
}

The basis lattice vectors of the simulation box are the function of $\zeta$ and $\eta$ in every timestep. We calculated the new lattice vectors at every $0.5 \mathrm{ps}$. The Verlet leapfrog integration method was applied. The timestep was $0.0005 \mathrm{ps}$ and the total simulation time was $12.5 \mathrm{ps}$, including $2.5 \mathrm{ps}$ for the equilibration time. The reported lattice constants herein were the average values obtained from 2.5 to $12.5 \mathrm{ps}$. The orthorhombic periodic boundary condition was used in the simulation.

The interatomic potential models for the aluminosilicate include the short-ranged pair potential and the long-ranged electrostatic force. We utilized two different potential models: one proposed by Winkler et al. ${ }^{9}$ (Winkler's potential) and the other by Matsui ${ }^{14}$ (Matsui's potential). In order to follow the potential models implemented in the DL_POLY package, we kept the proposed atom charges for the long-ranged electrostatic force but modified the proposed potential parameters into an equivalent one. For both the Winkler's potential and Matsui's potential, the long-ranged electrostatic force and the Born-Huggins-Meyer potential model were used. The potential form can be expressed as:

$U\left(r_{i j}\right)=\frac{q_{i} q_{j}}{r_{i j}}+A \exp \left[B\left(\alpha-r_{i j}\right)\right]-\frac{C}{r_{i j}{ }^{6}}$

where $r_{i j}$ is the distance between atoms $i$ and $j, q_{i}$ and $q_{j}$ the atom charges, and $A, B, \alpha$, and $C$ are the potential parameters, respectively. The potential parameters and the atom charges of the Winkler's and Matsui's potentials used herein are listed in Tables 1 and 2. It is worth noting that we did not include modification at a very short distance in the Winkler's potential. The rationale is that such modification should not affect the properties of the system at low and intermediate temperature. ${ }^{9}$ For all the short-ranged potentials, the cut-off distance was $6 \AA$. The standard Ewald sum method for the long-ranged electrostatic force was adapted with the cut-off distance of $6 \AA$.

The atom coordinates of mullite cannot be obtained directly from the inorganic crystal structure database (ICSD), because the occupancy factors of $\mathrm{Al} / \mathrm{Si}$ atoms at the $\mathrm{T}$ and $\mathrm{T}^{*}$ sites and $\mathrm{O}$ atom at the $\mathrm{O}(\mathrm{C})$ site do not equal to one. Alternatively, Angle and Prewitt ${ }^{15}$ suggested that it is possible to derive the mullite structure from sillimanite by removing $\mathrm{O}$ atoms at $\mathrm{O}(\mathrm{C})$ sites and meanwhile replacing twice of $\mathrm{Si}$ atoms by $\mathrm{Al}$. Most atomistic studies ${ }^{10,17,18}$ applied this rule to build the mullite model and it was consequently adapted herein. The mullite $18 \times 18 \times 6$ supercell was derived from $18 \times 18 \times 3$ sillimanite. Table 3 lists the crystallographic data of the sillimanite used in this study. The mullite structure was constructed by randomly removing oxygen vacancies at the $\mathrm{O}(\mathrm{C})$ sites and randomly replacing $\mathrm{Si}$ by $\mathrm{Al}$ in the supercell. A set of mullite models were created in which the $x$-values were equal to $0.20,0.25,0.30,0.40,0.50$, 
Table 2

Potential parameters and atom charges for the Matsui's potential

\begin{tabular}{|c|c|c|c|c|c|}
\hline Atom pair & $A\left(\mathrm{~kJ} \mathrm{~mol}^{-1}\right)$ & $B\left(\AA^{-1}\right)$ & $\alpha(\AA)$ & $C\left(\mathrm{~kJ} \mathrm{~mol}^{-1} \AA^{6}\right)$ & Charge $(e)$ \\
\hline $\mathrm{Al}-\mathrm{O}$ & 0.7196 & 5.8140 & 2.6067 & 3336.3000 & $\mathrm{Al}:+1.4175$ \\
\hline $\mathrm{Si}-\mathrm{O}$ & 0.6736 & 6.2112 & 2.5419 & 4467.1000 & $\mathrm{Si}:+1.8900$ \\
\hline $\mathrm{O}-\mathrm{O}$ & 1.1548 & 3.6232 & 3.6430 & 8210.2000 & O: -0.9450 \\
\hline
\end{tabular}

Table 3

Crystallographic data of sillimanite retrieved from ICSD database. The collection code is 25711 with the source originally from Burnham ${ }^{16}$

\begin{tabular}{llll}
\hline Atom & $X$ & $Y$ & $Z$ \\
\hline Al1 $(\mathrm{Al}(\mathrm{O}))$ & 0.0000 & 0.0000 & 0.0000 \\
A12 (Al(T)) & 0.1419 & 0.3447 & 0.2500 \\
Si1 $(\mathrm{Si})$ & 0.1535 & 0.3404 & 0.7500 \\
O1 $(\mathrm{O}(\mathrm{A}, \mathrm{B}))$ & 0.3599 & 0.4078 & 0.7500 \\
O2 $(\mathrm{O}(\mathrm{A}, \mathrm{B}))$ & 0.3576 & 0.4352 & 0.2500 \\
O3 $(\mathrm{O}(\mathrm{C}))$ & 0.4753 & 0.0008 & 0.7500 \\
O4 (O(D)) & 0.1248 & 0.2237 & 0.5164 \\
\hline
\end{tabular}

The space group is Pbnm with the lattice constants: $a=7.4856 \AA, b=7.6738 \AA$, and $c=5.7698 \AA$.

$0.55,0.60,0.66,0.70,0.75,0.80$, and 0.85 , respectively. The total number of atoms was 30,715 for $x=0.20$ and 29,452 for $x=0.85$. Since random distribution was involved in constructing the mullite, five samples were generated for each $x$-value. The data reported below were the average simulation results from these samples.

The random distribution of $\mathrm{Al} / \mathrm{Si}$ substitutions in the aforementioned $18 \times 18 \times 6$ supercell implies that there is no correlation of cation occupation at the $\mathrm{T}^{*}$ sites of mullite. Nevertheless, Angle and Prewitt ${ }^{15}$ pointed out that the $\mathrm{T}^{*}$ site is mostly occupied by Al. To cope with this observation, another set of mullite compositions were constructed in which the $\mathrm{T}^{*}$ sites were all occupied by Al. Since $\mathrm{O}(\mathrm{C})$ atom is cross-linked by alternating $\mathrm{Si}$ and $\mathrm{Al}$ in sillimanite, the complete occupation of $\mathrm{Al}$ at the $\mathrm{T}^{*}$ sites can be achieved by replacing one $\mathrm{Si}$ with $\mathrm{Al}$ linked with the oxygen vacancy. In order to maintain the charge balance, we randomly replaced another $\mathrm{Si}$ by $\mathrm{Al}$ in the supercell. We called the model with the complete $\mathrm{Al}$ occupation at the $\mathrm{T}^{*}$ sites a partially ordered model. Again for all the partially ordered models, five samples were generated for each $x$ and the average simulation results from these samples were obtained.

\section{Results}

The calculated lattice constants $a$ and $b$ from the models with randomly distributed and partially ordered $\mathrm{Al} / \mathrm{Si}$ substitutions are plotted in Fig. 1. The Matsui's potential was used. Both sets predicted that the lattice constant $a$ rises linearly while $b$ gradually decreases with increasing $x$-value. The trend predicted by the partially ordered set appeared to agree better with the experimental measurements. ${ }^{8}$ Fig. 2 plots the calculated lattice constants from the set of partially ordered models using the Matsui's and Winkler's potentials. Both interatomic potentials yielded the correct trend but that from the Matsui's appeared to agree better with the experimental measurements. Overall, the

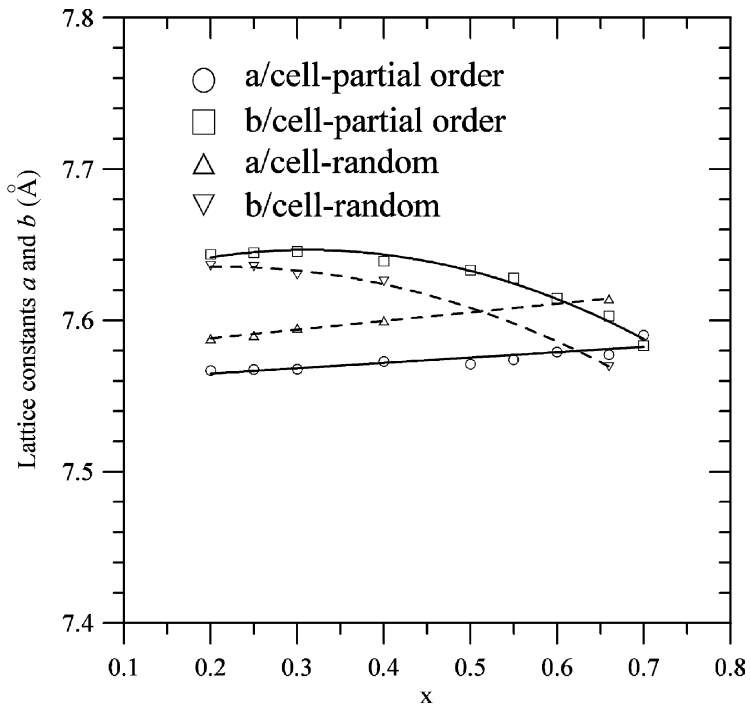

Fig. 1. Calculated lattice constants $a$ and $b$ from the models with randomly distributed and partially ordered Al/Si substitutions. The Matsui's potential was used.

best agreement between the calculated and experimental values was obtained when the partially ordered model with the Matsui's potential was used. Thus, if not particularly mentioned, all the subsequent simulation results were obtained from this combination.

Fig. 3 gives a detailed comparison between the calculated results and experimental data. All the five simulation results

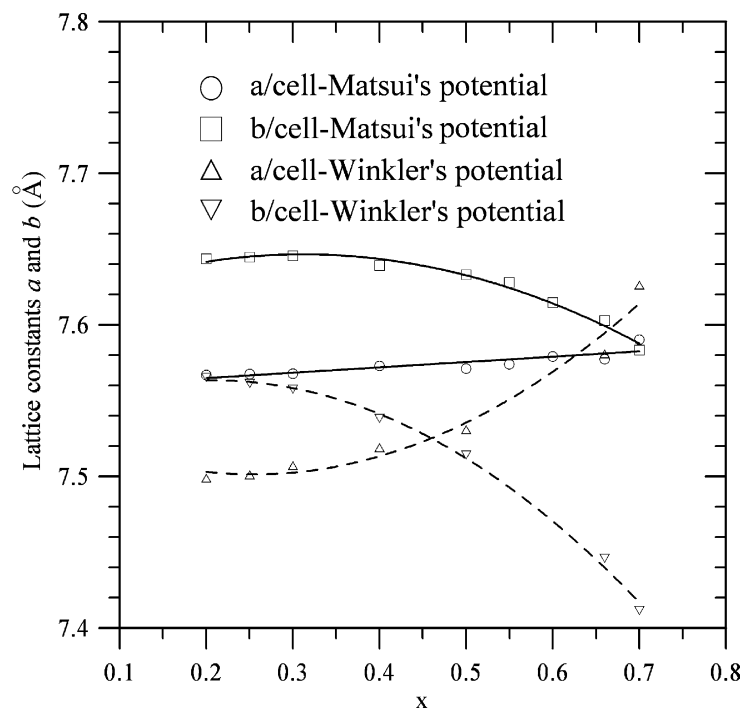

Fig. 2. Calculated lattice constants $a$ and $b$ from the partially ordered model using Matsui's and Winkler's potentials. 


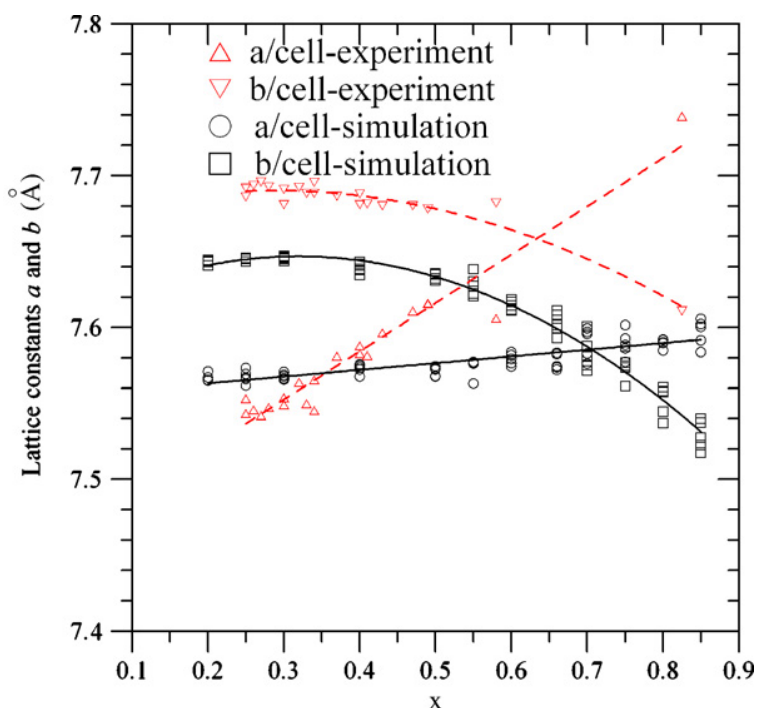

Fig. 3. Comparison of lattice constants $a$ and $b$ between the calculated and experimental values. The experimental data were obtained from Fischer and Schneider ${ }^{8}$ and the calculated data were based on the partially ordered model using the Matsui's potential.

for each $x$ were drawn. As shown in the figure, these simulation results were well-distributed within a satisfactory range for each $x$. The trend of the calculated lattice constant $a$ and $b$ was in good agreement with the experimental data. The curve of the calculated lattice constant $b$ was similar to that from experiments. Although the slope of the calculated lattice constant $a$ did not match well, the maximum mismatch between the calculated and experimental values was below $2 \%$. The interpolated crossing points were about 0.64 and 0.70 from experiments and simulation, respectively.

Fig. 4 shows the time evolution of the total static lattice energy, short-ranged potential and electrostatic energy per cell during the simulation. The stabilization of the static lattice energy was reached at about 2 ps. The contribution from the electrostatic force was greater than that from the short-ranged interatomic potential. Fig. 5 depicts the time evolution of lattice constants $a$ and $b$ during the simulation at the composition $x$ equal to $0.20,0.70$, and 0.85 , respectively. All the five simulation results for each $x$-value were drawn and the results were again well-distributed within a satisfactory range. The change of lattice constants in all compositions was stabilized at about $1 \mathrm{~ns}$. A common trend was observed for all the compositions in which the lattice constant $a$ increases while $b$ decreases from the initial sillimanite model. At the final equilibrated state, we observed that $a<b$ for $x=0.20, a \approx b$ for $x=0.70$ and $a>b$ for $x=0.85$. We also found (not shown) that the three inter-axial angles $\alpha, \beta$, and $\gamma$ during the simulation remained approximately at $90^{\circ}$. It thus indicated that the cell shape did not change with increasing $x$ during the simulation.

\section{Discussion}

All the aforementioned simulation results offer clear evidence that randomly distributed vacancies and $\mathrm{Al} / \mathrm{Si}$ substitutions produce different expansion-reducing effects in the $\mathbf{a}$ and $\mathbf{b}$
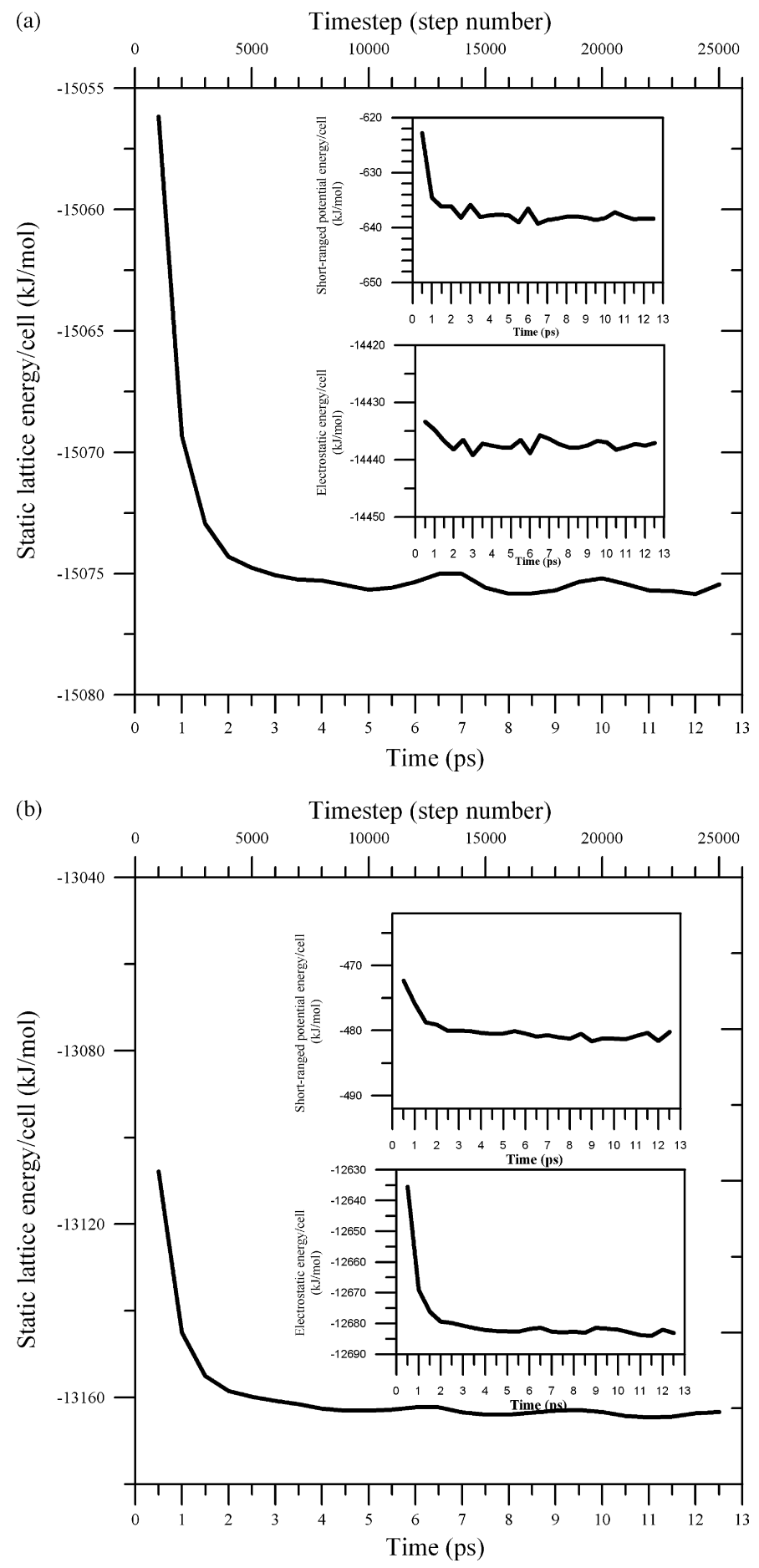

Fig. 4. Evolution of static lattice, short-ranged potential, and electrostatic energies for mullite composition at (a) $x=0.20$ and (b) $x=0.85$. The static lattice energy is the sum of the short-ranged potential energy (the upper side of the small figure) and the long-ranged electrostatic energy (the lower side of the small figure).

directions. Thus, the causes of lattice anomaly cannot be simply related to the possibility of structurally anisotropic distributions of oxygen vacancies suggested previously ${ }^{8}$. In order to probe the collective mechanisms from atoms and oxygen vacancies that would induce the anomaly of lattice constants in the $\left(\begin{array}{lll}0 & 0 & 1\end{array}\right)$ plane, bond length analysis and local atom displacement from 


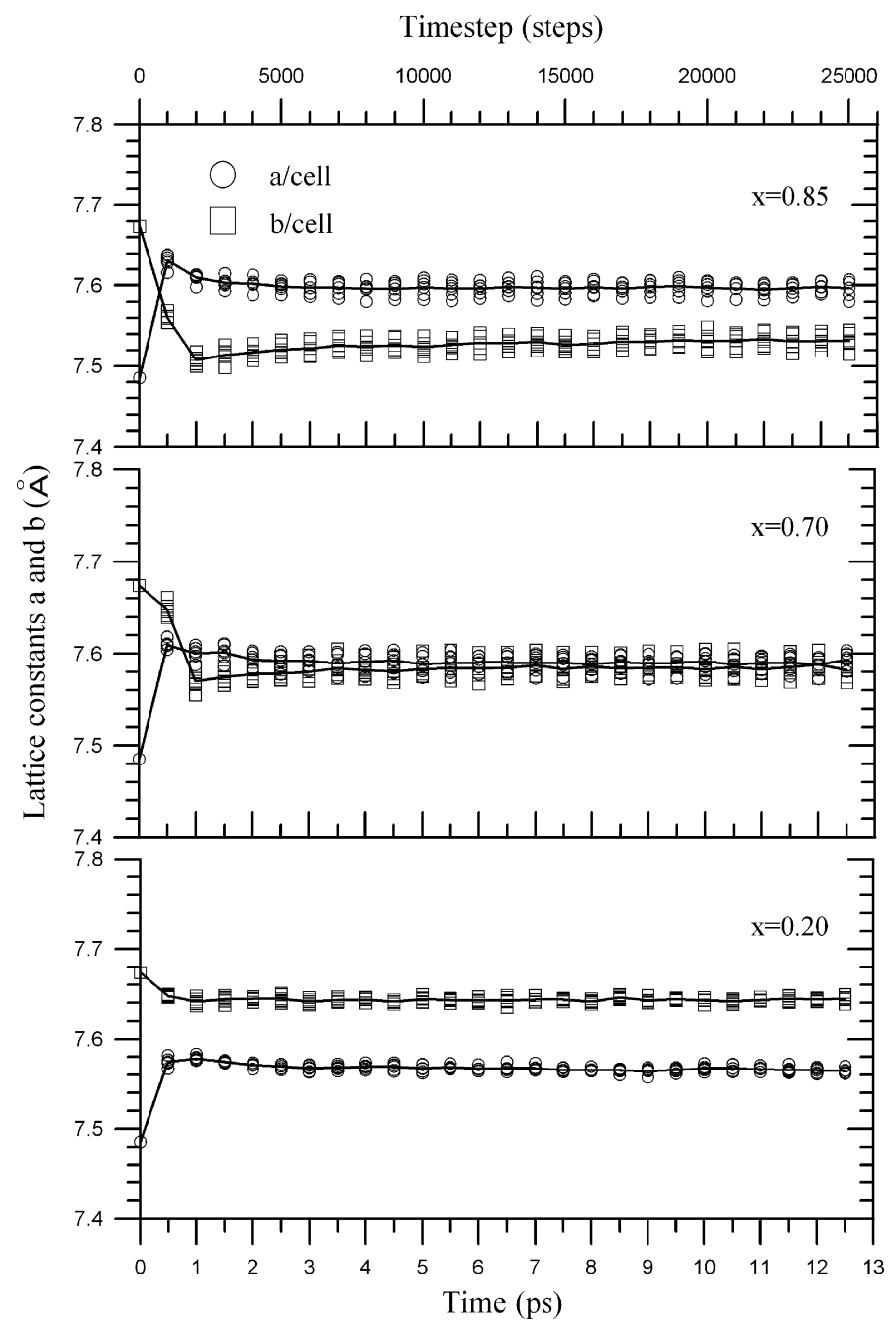

Fig. 5. Time-dependent evolution of calculated lattice constants $a$ and $b$ for mullite composition at $x=0.20,0.70$ and 0.85 .

oxygen vacancies were conducted. For the former, the bond length of each atom pair in tetrahedral elements within a cut-off of $2 \AA$ was counted. Table 4 lists the results at the composition $x$ equal to $0.20,0.70$, and 0.85 , respectively. We found that the bond length of $\mathrm{AlO}_{4}$ was longer than $\mathrm{SiO}_{4}$. The results support the expansion mechanism induced by $\mathrm{Al} / \mathrm{Si}$ substitutions. ${ }^{8}$ As the $x$ increases, the alumina contents increase as well and consequently, the lattice constants $a$ and $b$ increase.

Table 4

Tetrahedral bond lengths in mullite calculated for compositions $x=0.20,0.70$, and 0.85

\begin{tabular}{llll}
\hline Atom pair & \multicolumn{2}{l}{ Bond length $(\AA)$} \\
\cline { 2 - 4 } & $x=0.20$ & $x=0.70$ & $x=0.85$ \\
\hline $\mathrm{Al}(\mathrm{T})-\mathrm{O}(\mathrm{A}, \mathrm{B})$ & 1.76 & 1.76 & 1.77 \\
$\mathrm{Al}(\mathrm{T})-\mathrm{O}(\mathrm{D})$ & 1.76 & 1.76 & 1.76 \\
$\mathrm{Al}(\mathrm{T})-\mathrm{O}(\mathrm{C})$ & 1.76 & 1.76 & 1.77 \\
$\mathrm{Si}-\mathrm{O}(\mathrm{A}, \mathrm{B})$ & 1.64 & 1.64 & 1.65 \\
$\mathrm{Si}-\mathrm{O}(\mathrm{D})$ & 1.64 & 1.64 & 1.65 \\
$\mathrm{Si}-\mathrm{O}(\mathrm{C})$ & 1.64 & 1.64 & 1.65 \\
\hline
\end{tabular}
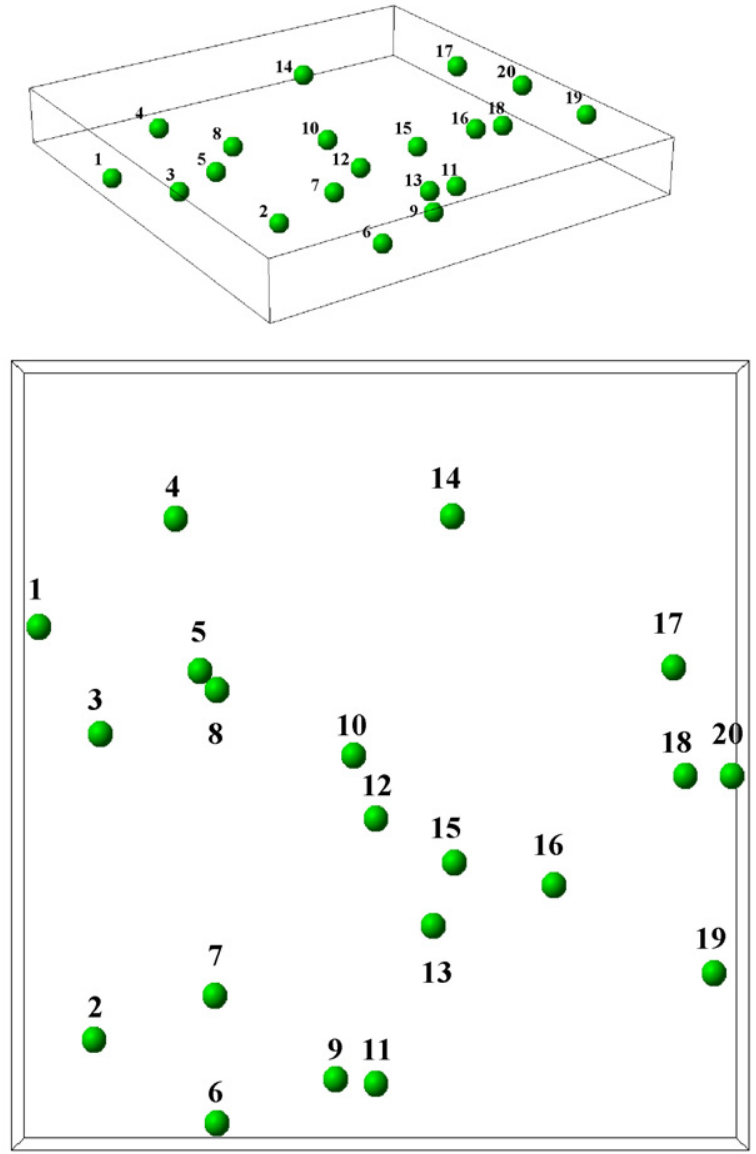

Fig. 6. Distribution of 20 isolated oxygen vacancies in the mullite $18 \times 18 \times 6$ supercell.

Analysis of local atom displacement from the oxygen vacancy provides another mean to examine possible lattice contraction and expansion mechanisms. For mullite, we are particularly interested in studying such displacement as the $x$-value increases. To this end, we re-designed the mullite $18 \times 18 \times 6$ supercell for $x=0.20$ and 0.40 . The positions of 20 isolated oxygen vacancies in the model were fixed a priori. Fig. 6 depicts and labels these designated oxygen vacancies in the supercell. These vacancies were served as the seeds to probe the local expansion and contraction when the $x$-value changed from 0.20 to 0.40 . Other remaining oxygen vacancies and $\mathrm{Al} / \mathrm{Si}$ substitutions were generated randomly outside the cut-off radius $4 \AA$ of the designated vacancies. Doing so thus ensures that for these seeds, the topology of their neighborhood atom arrangement remains almost unchanged in about the size of a unit cell in the $\left(\begin{array}{ll}0 & 0 \\ 1\end{array}\right)$ plane. Under this controlled circumstance, it is then possible to compare the local atom displacement within the cut-off of the designated vacancy as the $x$-value increases.

The relationship between local atom displacement and lattice expansion and contraction can be understood as follows. Consider a simple illustrative example in Fig. 7 in which an atom is moving away from the designated vacancy as the $x$-value changes from 0.20 to 0.40 . Because the relative displacement is quite small, the atom is likely to stay in the same quadrant in both configurations. Under this assumption, the relative displacement 


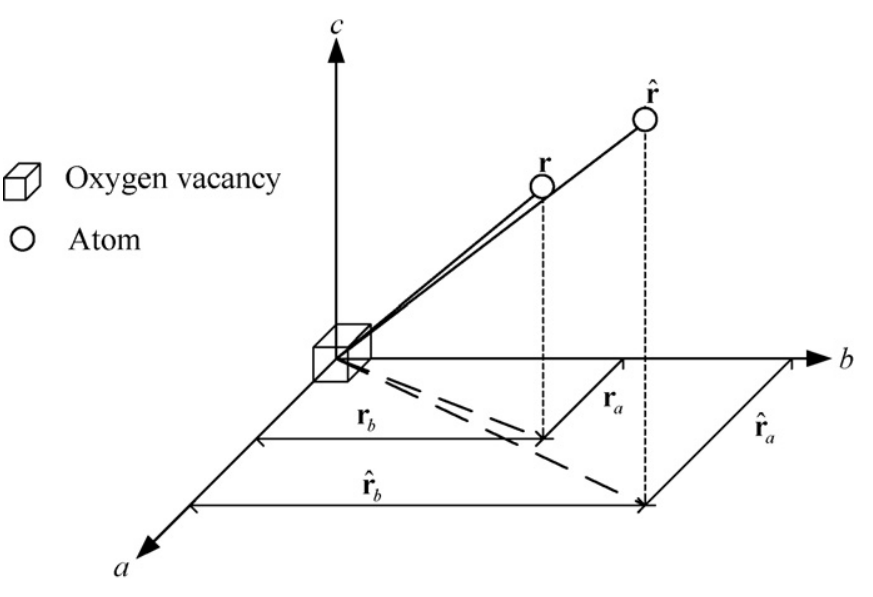

Fig. 7. Illustration of an atom moving away from an oxygen vacancy.
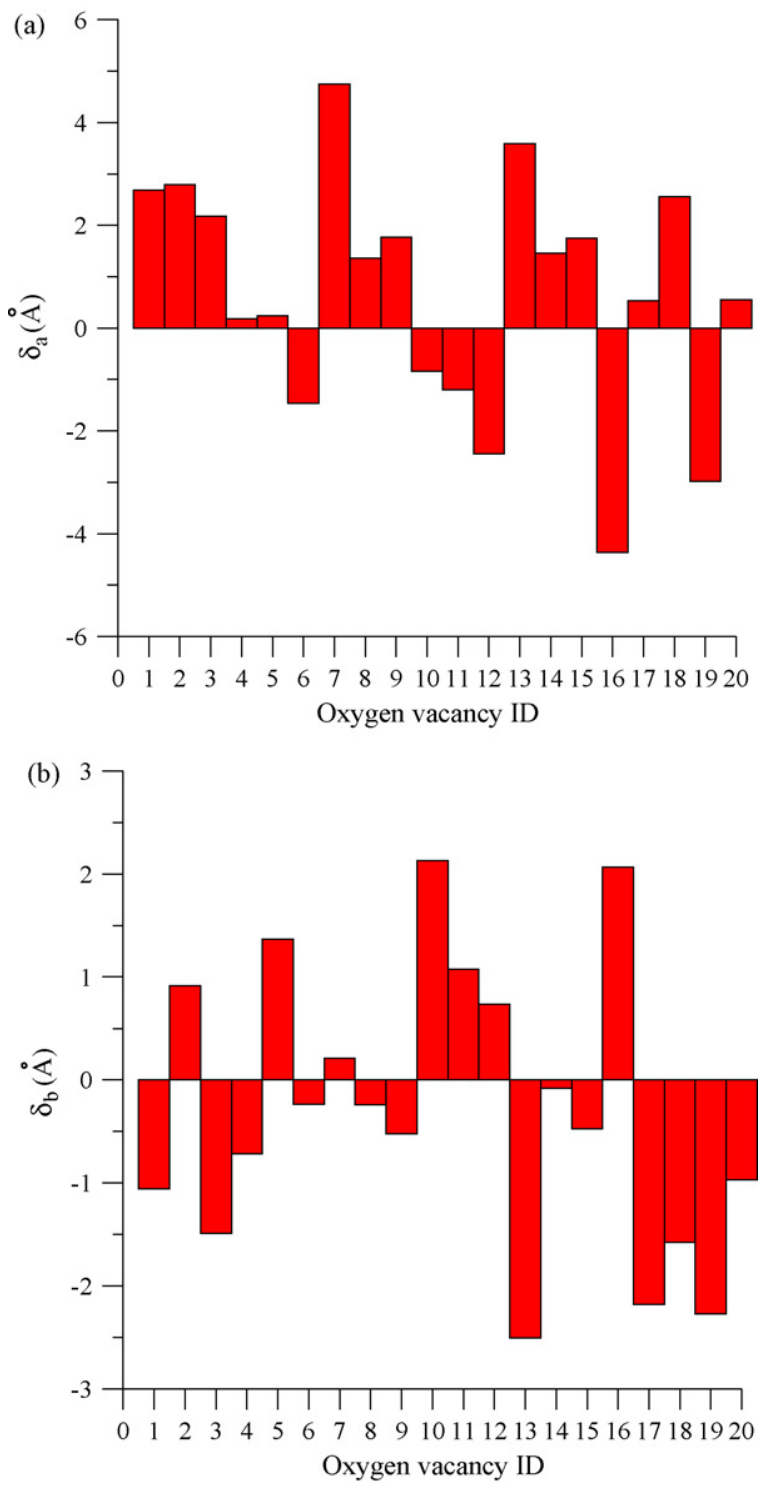

Fig. 8. Contribution of local atom displacement in the neighborhood of designated oxygen vacancies along (a) the $a$-axis and (b) the $b$-axis. The oxygen vacancy ID is listed in Fig. 6. $\delta_{a}$ of an atom in the a direction with respect to the designated vacancy can be expressed as:

$\delta_{a}=\operatorname{sgn}\left(\mathbf{r} \cdot \mathbf{e}_{a}\right)(\hat{\mathbf{r}}-\mathbf{r}) \cdot \mathbf{e}_{a}$

in which $\mathbf{r}$ and $\hat{\mathbf{r}}$ are the position vectors of the atom with respect to the vacancy at $x$ equal to 0.20 and 0.40 , respectively, and $\mathbf{e}_{a}$ is the unit vector in the a direction. The sgn in Eq. (2) is the sign function that yields +1.0 if $\mathbf{r} \cdot \mathbf{e}_{a}>0$ and -1.0 otherwise. Similarly, the relative movement $\delta_{b}$ of an atom in the $\mathbf{b}$ direction with respect to the designated vacancy can be expressed as:

$\delta_{b}=\operatorname{sgn}\left(\mathbf{r} \cdot \mathbf{e}_{b}\right)(\hat{\mathbf{r}}-\mathbf{r}) \cdot \mathbf{e}_{b}$

in which $\mathbf{e}_{b}$ is the unit vector in the $\mathbf{b}$ direction. The positive values of $\delta_{a}$ and $\delta_{b}$ stand for expanding action in the neighborhood of the vacancy while the negative ones for contracting action. Every atom in the neighborhood of the designated vacancy would result in some contributions of expanding or contracting action in the $\mathbf{a}$ and $\mathbf{b}$ directions. Fig. 8 plots the summation of such action for all the 20 oxygen vacancies. As shown in the figure, 14 sites sensed expansion and 6 sites sensed contraction in the a direction. The numbers changed to 7 and 13 in the $\mathbf{b}$ direction. The total summation was $13.1 \AA$ in the a direction and $-5.8 \AA$ in the $\mathbf{b}$ direction.

The results shown in Fig. 8 were rather inhomogeneous. All the four possible combinations of lattice contraction and expansion in the $\mathbf{a}$ and $\mathbf{b}$ directions were sensed by some vacancies. The results suggest that such local contraction and expansion are rather complex as the $x$-value changes from 0.20 to 0.40 . Overall, anisotropic atom displacement is induced in the a and $\mathbf{b}$ directions and such action supports the anomalous trend where the lattice constant $a$ increases and $b$ decreases as the $x$-value increases. Additionally, this induced anisotropic atom displacement offers another evidence that randomly distributed vacancies and $\mathrm{Al} / \mathrm{Si}$ substitutions are capable of producing different expansion-reducing effects in the $\mathbf{a}$ and $\mathbf{b}$ directions.

\section{Conclusions}

The anomaly of $a$ and $b$ lattice constants with increasing $x$ value of mullite was successfully predicted using the constant stress molecular dynamics method. The best agreement between the calculated and experimental values was obtained when the partially ordered model with the Matsui's potential was used. The maximum mismatch between the calculated and experimental values was below $2 \%$. Bond length analysis of tetrahedral elements supports the expansion mechanism induced by $\mathrm{Al} / \mathrm{Si}$ substitutions. Anisotropic atom displacement sensed by the designated oxygen vacancies supports the anomalous trend where the lattice constant $a$ increases and $b$ decreases as the $x$-value increases.

The present study offers clear evidence that the causes of lattice anomaly are tightly interwoven with the coexistence of the formation of oxygen vacancies and $\mathrm{Al} / \mathrm{Si}$ substitutions. The anomaly cannot be simply related to the hypothesis associated with the possibility of structurally anisotropic distributions of oxygen vacancies. 


\section{Acknowledgements}

The research was supported by the National Science Council in Taiwan under award no. 94-2218-E-002-062. We are grateful to the National Center for High-performance Computing for providing the computational resources. Jen-Chang Chen would also like to thank Dr. Bill Smith at Daresbury Laboratory for the valuable discussion on the DL_POLY program.

\section{References}

1. Schneider, H., Basic properties of mullite. In Mullite, ed. H. Schneider and S. Komarneni. Wiley-VCH, Weinheim, 2005, pp. 141-155.

2. Cameron, W. E., Mullite: a substituted alumina. Am. Mineral., 1977, 62, 747-755.

3. Fischer, R. X., Schneider, H. and Schmücker, M., Crystal structure of Al-rich mullite. Am. Mineral., 1994, 79, 983-990.

4. Fischer, R. X., Schneider, H. and Voll, D., Formation of aluminum rich 9:1 mullite and its transformation to low alumina mullite upon heating. J. Eur. Ceram. Soc., 1996, 16, 109-113.

5. Cameron, W. E., Composition and cell dimensions of mullite. Am. Mineral., 1977, 62, 747-755.

6. Schneider, H., Fischer, R. X. and Voll, D., Mullite with lattice constants $a>b$. J. Am. Ceram. Soc., 1993, 76, 1879-1881.

7. Schneider, H. and Rymon-Lipinski, T., Occurrence of pseudotetragonal mullite. J. Am. Ceram. Soc., 1988, 71, c161-c164.
8. Fischer, R. X. and Schneider, H., Crystal chemistry of mullite and related phases. In Mullite, ed. H. Schneider and S. Komarneni. Wiley-VCH, Weinheim, 2005, pp. 1-140.

9. Winkler, A., Horbach, J., Kob, W. and Binder, K., Structure and diffusion in amorphous aluminum silicate: a molecular dynamics computer simulation. J. Chem. Phys., 2004, 120, 384-393.

10. Lacks, D. J., Hildmann, B. and Schneider, H., Effects of disorder in mullite: molecular dynamics simulation and energy landscape analysis. Phys. Rev. $B, 2005,72,214305$.

11. Smith, W. and Forester, T., DL_POLY_2.0: a general-purpose parallel molecular dynamics simulation package. J. Mol. Graphics, 1996, 14, 136-141.

12. Parrinello, M. and Rahman, A., Polymorphic transitions in single crystals: a new molecular dynamics method. J. Appl. Phys., 1981, 52, 7182-7190.

13. Melchionna, S., Ciccotti, G. and Holian, B. L., Hoover NPT dynamics for systems varying in shape and size. Mol. Phys., 1993, 78, 533-544.

14. Matsui, M., Molecular dynamics study of the structures and bulk moduli of crystals in the system $\mathrm{CaO}-\mathrm{MgO}-\mathrm{Al}_{2} \mathrm{O}_{3}-\mathrm{SiO}_{2}$. Phys. Chem. Miner., 1996, 23, 345-353.

15. Angel, R. J. and Prewitt, C. T., Crystal structure of mullite: a re-examination of the average structure. Am. Mineral., 1986, 71, 1476-1482.

16. Burnham, C. W., Refinement of the crystal structure of sillimanite. Z. Kristallogr., 1963, 118, 127-148.

17. Wondraczek, L., Heide, G., Kilo, M., Nedeljkovic, N., Borchardt, G. and Jackson, R. A., Computer simulation of defect structure in sillimanite and mullites. Phys. Chem. Miner., 2002, 29, 341-345.

18. Padlewski, S., Heine, H. and Price, G. D., A microscopic model for a very stable incommensurate modulated mineral: mullite. J. Phys.: Condens. Matter, 1993, 5, 3417-3430. 